\title{
Consistency and its Converse for Roommate Markets
}

\author{
Bettina Klaus*
}

March 13, 2017

\begin{abstract}
For classical marriage markets with equal numbers of men and women and where all men find all women acceptable and all women find all men acceptable, Sasaki and Toda (1992) characterized the core by same-side anonymity for marriage markets, Pareto optimality, consistency, and converse consistency. Nizamogullari and Özkal-Sanver (2014) generalized this result to the domain of classical marriage markets by adding individual rationality and replacing same-side anonymity for marriage markets with a stronger property called gender fairness. We generalize both results by characterizing the core on the domain of solvable roommate markets without socalled "3-rings" (and on the domain of marriage markets) by individual rationality, anonymity, Pareto optimality, consistency, and converse consistency. We also prove that extending this characterization to the domain of solvable roommate markets is not possible.
\end{abstract}

JEL classification: C78, D63.

Keywords: Converse Consistency, Core, Marriage and Roommate Markets.

\section{Introduction}

We consider one-to-one matching markets in which agents can either be matched as pairs or remain single. These markets are known as roommate markets and they include, as special cases, the wellknown marriage markets (Gale and Shapley, 1962, Roth and Sotomayor, 1990). Furthermore, a roommate market is a simple example of hedonic coalition formation as well as network formation: in a "roommate coalition" situation, only coalitions of size one or two can be formed and in a "roommate network" situation, each agent is allowed or able to form only one link (for surveys of coalition and network formation see Demange and Wooders, 2004; Jackson, 2008). We will refer to marriage markets as introduced by Gale and Shapley (1962) as classical marriage markets. The reason for this terminology is that we will embed marriage markets into the more general roommate market model by restricting agents' preferences (same-gender agents are unacceptable) instead of feasibility constraints (same-gender agents cannot be matched).

Various characterizations of the core have been established for classical marriage markets (Sasaki and Toda, 1992, Toda, 2006). For these well-known "benchmark matching markets," the set of

${ }^{*}$ Faculty of Business and Economics, University of Lausanne, Internef 538, CH-1015, Lausanne, Switzerland; email: bettina.klaus@unil.ch. I thank two referees, Burak Can, Mehmet Karakaya, Panos Protopapas, and Jan Christoph Schlegel for their valuable comments and interesting discussions. Special thanks go to an advisory editor at Games and Econoic Behavior for giving detailed feedback and pointing out an omission in the original version of this article; her/his comments helped considerably improve this article. I gratefully acknowledge financial support from the Swiss National Science Foundation (SNSF). 
core matchings forms a distributive lattice which reflects polarization between the two sides of the market (Knuth, 1997, attributed this result to John Conway). For one-sided matching markets such as roommate markets and coalition/network formation, the core may be empty. Furthermore, for a one-sided matching market with a non-empty core, which we refer to as solvable, the core does not exhibit such strong structural properties and hence it is an interesting question to ask if the structure of the core drives the normative characteristics of the core or if the characterizing properties are strong enough independent of the particular (lattice) structure of the core.

Toda (2006) provided two characterizations of the core using the well-known solidarity property of population monotonicity, which he adapted to the two-sided setup of classical marriage markets. Klaus (2011) and Can and Klaus (2013) introduced the new properties of competition and resource sensitivity for roommate markets to account for the loss of two-sidedness and established associated core characterizations for various roommate market preference domains (including the domain of marriage markets). However, whether or not the very first characterization of the core for classical marriage markets (Sasaki and Toda, 1992) could also be extended to the one-sided model of roommate markets has been an open question for twenty five years. We provide the answer to this question in this article.

For classical marriage markets with equal numbers of men and women and where all men find all women acceptable and all women find all men acceptable, Sasaki and Toda $(1992)$ characterized the core by same-side anonymity for marriage markets 11 Pareto optimality, consistency ${ }^{2}$ and converse consistency ${ }^{3}$. This characterization is very appealing for its use of various normative requirements that are used in many economic contexts: anonymity is a commonly used fairness property, Pareto optimality is a versatile ordinal efficiency requirement, consistency is a condition of "procedural stability" when the set of agents may change, and converse consistency has practical appeal whenever small markets are much easier to solve than large ones.

Nizamogullari and Özkal-Sanver (2014) generalized this result to the domain of classical marriage markets by adding individual rationality and replacing same-side anonymity for marriage markets with a stronger property called gender fairness 4 Furthermore, Özkal-Sanver 2010, Proposition 4.2) showed that on the domain of all roommate markets, no solution satisfies Pareto optimality, anonymity $5^{5}$ and converse consistency. However, the proof of this impossibility result used an unsolvable roommate market. Hence, the question whether or not the characterization of Sasaki and Toda (1992) can be extended to the domain of solvable roommate markets or to any of its subdomains has not been answered until now.

In this article, we first show that on the domain of solvable roommate markets, the core does not satisfy converse consistency (Proposition 2). Second, we extend the characterizations of Sasaki and Toda (1992) and Nizamogullari and Özkal-Sanver (2014) to the domain of solvable roommate markets without so-called "3-rings" (Theorem 1): a solution satisfies individual rationality, anonymity,

\footnotetext{
${ }^{1}$ Same-side anonymity for marriage markets: matchings assigned by the solution do not depend on agents' names when the men's names are permuted on the men's side of the market or when the women's names are permuted on the women's side of the market.

${ }^{2}$ Consistency: if a set of matched agents leaves, then the solution should still match the remaining agents as before.

${ }^{3}$ Converse consistency: matchings assigned by the solution are (conversely) related to the matchings the solution assigns to certain restricted roommate markets (with at most four agents).

${ }^{4}$ Gender fairness (Özkal-Sanver, 2004): for marriage markets with an equal number of men and women, renaming men as women, and renaming women as men, does not change the outcomes chosen by the solution when taking the renaming of agents into account.

${ }^{5}$ Anonymity: matchings assigned by the solution do not depend on agents' names.
} 
Pareto optimality, consistency, and converse consistency if and only if it is the core. We also show that the same properties characterize the core for marriage markets (Theorem 2). Third, we demonstrate that the properties of Theorem 1 are compatible on the domain of solvable roommate markets by constructing a conversely consistent extension of the core (Proposition 3).

Our paper is organized as follows. In Section 2 we present the roommate market model, basic properties of solutions, and the core. In Section 3, we introduce the variable population properties consistency and converse consistency; we also show that on the domain of solvable roommate markets, the core does not satisfy converse consistency (Proposition 2). Section 4 first, for classical marriage markets, reviews the above mentioned characterizations of the core (Theorems A and B) and an impossibility result (Ö̈zkal-Sanver, 2010, Proposition 4.2). We then establish new characterizations of the core for solvable roommate markets without 3-rings (Theorem 1) and for marriage markets (Theorem 2) and a new possibility result on the domain of solvable roommate markets (Proposition 3).

\section{2 (Roommate) Markets}

The following Subsections 2.1 and 2.2 mostly follow Klaus (2011) and Can and Klaus (2013).

\subsection{The Model}

We consider Gale and Shapley]s (1962, Example 3) roommate markets with variable sets of agents, e.g., the allocation of dormitory rooms at a university occurring every year for different sets of students.

Let $\mathbb{N}$ be the set of potential agent: $\sqrt{6}$ and $\mathcal{N}$ be the set of all non-empty finite subsets of $\mathbb{N}$, i.e., $\mathcal{N}=\{N \subset \mathbb{N}|\infty>| N \mid>0\}$. For $N \in \mathcal{N}, L(N)$ denotes the set of all linear orders over $N \mid 7$ For $i \in N$, we interpret $R_{i} \in L(N)$ as agent $i$ 's strict preferences over sharing a room (being matched) with any of the agents in $N \backslash\{i\}$ and being single (consuming an outside option); e.g., $R_{i}: j, k, i, l$ means that $i$ would first like to share a room with $j$, then with $k$, and then $i$ would prefer to stay alone to sharing the room with $l$. If $j P_{i} i$, then agent $i$ finds agent $j$ acceptable and if $i P_{i} j$, then agent $i$ finds agent $j$ unacceptable. $\mathcal{R}^{N}=\prod_{N} L(N)$ denotes the set of all preference profiles of agents in $N$ (over agents in $N$ ). A (roommate) market consists of a set of agents $N \in \mathcal{N}$ and their preferences $R \in \mathcal{R}^{N}$ and is denoted by $(N, R)$. A marriage market is a market $(N, R)$ such that $N$ is the union of two disjoint sets $M$ and $W$ and each agent in $M$ (respectively $W$ ) prefers being single to being matched with any other agent in $M$ (respectively $W$ ).

A matching $\mu$ for market $(N, R)$ is a function $\mu: N \rightarrow N$ of order two, i.e., for all $i \in N$, $\mu(\mu(i))=i$. Thus, at any matching $\mu$, the set of agents is partitioned into pairs of agents who share a room and singletons (agents who do not share a room). Agent $\mu(i)$ is agent $i$ 's match and if $\mu(i)=i$ then $i$ is matched to himself or single. For notational convenience, we often denote a matching in terms of the induced partition, e.g., for $N=\{1,2,3,4,5\}$ and matching $\mu$ such that

\footnotetext{
${ }^{6}$ All results remain valid for a finite set of potential agents that contains at least 8 agents (the proof of our main result, Theorem 1, as well as the independence of its properties can be shown with a population of 8 agents).

${ }^{7} \mathrm{~A}$ linear order over $N$ is a binary relation $\bar{R}$ that satisfies antisymmetry (for all $i, j \in N$, if $i \bar{R} j$ and $j \bar{R} i$, then $i=j$ ), transitivity (for all $i, j, k \in N$, if $i \bar{R} j$ and $j \bar{R} k$, then $i \bar{R} k$ ), and comparability (for all $i, j \in N, i \bar{R} j$ or $j \bar{R} i$ ). By $\bar{P}$ we denote the asymmetric part of $\bar{R}$. Hence, given $i, j \in N, i \bar{P} j$ means that $i$ is strictly preferred to $j ; i \bar{R} j$ means that $i \bar{P} j$ or $i=j$ and that $i$ is weakly preferred to $j$.
} 
$\mu(1)=2, \mu(3)=3$ and $\mu(4)=5$ we write $\mu=\{(1,2), 3,(4,5)\}$. For $S \subseteq N$, we denote by $\mu(S)$ the set of agents that are matched to agents in $S$, i.e., $\mu(S)=\{i \in N \mid \mu(i) \in S\}$. We denote the set of matchings for market $(N, R)$ by $\mathcal{M}(N)$ (note that the set of matchings does not depend on preferences $R$ ). If it is clear which market $(N, R)$ we refer to, matchings are assumed to be elements of $\mathcal{M}(N)$. Since agents only care about their own matches, we derive agents' preferences over matchings as follows: for all agents $i \in N$ and matchings $\mu, \mu^{\prime} \in \mathcal{M}(N), \mu(i) R_{i} \mu^{\prime}(i)$ if and only if $\mu R_{i} \mu^{\prime}: 8$

Given a market $(N, R)$ and $N^{\prime} \subseteq N$, we define the reduced preferences $R^{\prime} \in \mathcal{R}^{N^{\prime}}$ of $R$ to $N^{\prime}$ as follows:

(i) for all $i \in N^{\prime}, R_{i}^{\prime} \in L\left(N^{\prime}\right)$ and

(ii) for all $j, k, l \in N^{\prime}, j R_{l}^{\prime} k$ if and only if $j R_{l} k$.

We denote the reduced preferences of $R$ to $N^{\prime}$ by $R_{N^{\prime}}$.

Given a market $(N, R)$, a matching $\mu \in \mathcal{M}(N)$, and $N^{\prime} \subseteq N$ such that $\mu\left(N^{\prime}\right)=N^{\prime}$, the reduced (roommate) market of $(N, R)$ at $\mu$ to $N^{\prime}$ equals $\left(N^{\prime}, R_{N^{\prime}}\right)$ and we define the reduced matching $\mu_{N^{\prime}}$ of $\mu$ to $N^{\prime}$ as follows:

(i) $\mu_{N^{\prime}}: N^{\prime} \rightarrow N^{\prime}$ and

(ii) for all $i \in N^{\prime}, \mu_{N^{\prime}}(i)=\mu(i)$.

Note that $\mu_{N^{\prime}} \in \mathcal{M}\left(N^{\prime}\right)$.

In the sequel, we consider various domains of markets: the domain of all markets $\mathfrak{D}$, the domain of marriage markets $\mathfrak{D}_{M}$, and later the domains of solvable markets, of solvable markets without 3 -rings, and of no odd rings markets. To avoid notational complexity when introducing solutions and their properties, we use the domain of all markets $\mathfrak{D}$ with the understanding that any other domain could be used as well.

A solution $\varphi$ on $\mathfrak{D}$ is a correspondence that associates with each market $(N, R) \in \mathfrak{D}$ a nonempty subset of matchings, i.e., for all $(N, R) \in \mathfrak{D}, \varphi(N, R) \subseteq \mathcal{M}(N)$ and $\varphi(N, R) \neq \emptyset$. A subsolution $\psi$ of $\varphi$ on $\mathfrak{D}$ is a correspondence that associates with each market $(N, R) \in \mathfrak{D}$ a nonempty subset of matchings in $\varphi(N, R)$, i.e., for all markets $(N, R) \in \mathfrak{D}, \psi(N, R) \subseteq \varphi(N, R)$ and $\psi(N, R) \neq \emptyset$. A proper subsolution $\psi$ of $\varphi$ on $\mathfrak{D}$ is a subsolution of $\varphi$ on $\mathfrak{D}$ such that $\psi \neq \varphi$.

\subsection{Basic Properties and the Core}

We first introduce a voluntary participation condition based on the idea that no agent can be forced to share a room.

Definition (Individual Rationality). Let $(N, R) \in \mathfrak{D}$ and $\mu \in \mathcal{M}(N)$. Then, $\mu$ is individually rational if for all $i \in N, \mu(i) R_{i} i$. IR $(N, R)$ denotes the set of all these matchings. A solution $\varphi$ on $\mathfrak{D}$ is individually rational if it only assigns individually rational matchings, i.e., for all $(N, R) \in \mathfrak{D}$, $\varphi(N, R) \subseteq I R(N, R)$.

\footnotetext{
${ }^{8}$ Note that when extending preferences from an agent's matches to matchings, antisymmetry is lost because an agent will always be indifferent between two matchings that assign him the same match.
} 
An individually rational matching for a marriage market $(N, R) \in \mathfrak{D}_{M}$ respects the partition of agents into two types and never matches two men or two women. Hence, we embed marriage markets into our roommate market framework by an assumption on preferences (same-gender agents are unacceptable) and individual rationality to ensure that no two agents of the same gender are matched. We refer to a marriage market for which matching agents of the same gender is not feasible as a classical marriage market (Gale and Shapley, 1962).

Anonymity requires that the agents' matches do not depend on their names. A permutation $\pi$ of $\mathbb{N}$ is a bijective function $\pi: \mathbb{N} \rightarrow \mathbb{N}$. By $\Pi^{\mathbb{N}}$ we denote the set of all permutations of $\mathbb{N}$.

Let $N \in \mathcal{N}$. Given $\pi \in \Pi^{\mathbb{N}}$ and a market $(N, R)$, let $N^{\pi}=\left\{i \in \mathbb{N} \mid \pi^{-1}(i) \in N\right\}$ and $R^{\pi} \in \mathcal{R}^{N^{\pi}}$ be such that for all $i, j, k \in N, i P_{j}^{\pi} k$ if and only if $\pi^{-1}(i) P_{\pi^{-1}(j)} \pi^{-1}(k)$. Furthermore, for $\mu \in \mathcal{M}(N)$ let $\mu^{\pi} \in \mathcal{M}\left(N^{\pi}\right)$ be such that for all $i \in N^{\pi}, \mu^{\pi}(i)=\pi\left(\mu\left(\pi^{-1}(i)\right)\right)$.

Definition (Anonymity). A solution $\varphi$ on $\mathfrak{D}$ is anonymous if for all $\pi \in \Pi^{\mathbb{N}}$ and all $(N, R) \in \mathfrak{D}$, $\mu \in \varphi(N, R)$ and $\left(N^{\pi}, R^{\pi}\right) \in \mathfrak{D}$ imply $\mu^{\pi} \in \varphi\left(N^{\pi}, R^{\pi}\right)$.

Next, we introduce the well-known condition of Pareto optimality.

Definition (Pareto Optimality). Let $(N, R) \in \mathfrak{D}$ and $\mu \in \mathcal{M}(N)$. Then, $\mu$ is Pareto optimal if there is no other matching $\mu^{\prime} \in \mathcal{M}(N)$ such that for all $i \in N, \mu^{\prime} R_{i} \mu$ and for some $j \in N, \mu^{\prime} P_{j} \mu$. $P O(N, R)$ denotes the set of all these matchings. A solution $\varphi$ on $\mathfrak{D}$ is Pareto optimal if it only assigns Pareto optimal matchings, i.e., for all $(N, R) \in \mathfrak{D}, \varphi(N, R) \subseteq P O(N, R)$.

A matching $\mu$ for market $(N, R) \in \mathfrak{D}$ is blocked by a pair $\{i, j\} \subseteq N$ [possibly $i=j$ ] if $j P_{i} \mu(i)$ and $i P_{j} \mu(j)$. If $\{i, j\}$ blocks $\mu$, then $\{i, j\}$ is called a blocking pair for $\mu$. A matching is individually rational if there is no blocking pair $\{i, j\}$ with $i=j$.

Definition (Stability and Solvability). Let $(N, R) \in \mathfrak{D}$ and $\mu \in \mathcal{M}(N)$. Then, $\mu$ is stable if there is no blocking pair for $\mu . S(N, R)$ denotes the set of all these matchings. A market is solvable if stable matchings exist, i.e., $(N, R)$ is solvable if and only if $S(N, R) \neq \emptyset$. The domain of solvable markets is denoted by $\mathfrak{D}_{S}$. Furthermore, on the domain of solvable markets $\mathfrak{D}_{S}$ and all its subdomains, a solution $\varphi$ is stable if it only assigns stable matchings, i.e., for all $(N, R) \in \mathfrak{D}_{S}$, $\varphi(N, R) \subseteq S(N, R) \neq \emptyset$.

Gale and Shapley (1962) showed that all classical marriage markets are solvable and they gave an example of an unsolvable market (Gale and Shapley, 1962, Example 3).

Chung (2000) introduced a sufficient condition for solvability that also applies to the larger domain of weak preferences. We formulate his well-known no odd rings condition for our strict preference setup while keeping the name no odd rings condition.

Let $(N, R) \in \mathfrak{D}$. Then, a ring for market $(N, R)$ is an ordered subset of agents $\left\{i_{1}, i_{2}, \ldots, i_{k}\right\} \subseteq$ $N, k \geq 3$, such that for all $t \in\{1,2, \ldots, k\}, i_{t+1} P_{i_{t}} i_{t-1} P_{i_{t}} i_{t}$ (subscript modulo $k$ ). If $k$ is odd, then $\left\{i_{1}, i_{2}, \ldots, i_{k}\right\}$ is an odd ring for market $(N, R)$. A market $(N, R) \in \mathfrak{D}$ is a no odd rings market if there exists no odd ring in $(N, R)$. The domain of all such markets is called the domain of no odd rings markets and denoted by $\mathfrak{D}_{N O R}$. We will obtain our main result (Theorem 1) for a domain that lies in between the domain of solvable markets and the domain of no odd ring markets: a solvable market $(N, R) \in \mathfrak{D}_{S}$ for which there exists no 3-ring is a solvable market without 3-rings. The domain of all such markets is called domain of solvable markets without 3-rings and denoted by $\mathfrak{D}_{S N 3 R}$. We have $\mathfrak{D} \supsetneq \mathfrak{D}_{S} \supsetneq \mathfrak{D}_{S N 3 R} \supsetneq \mathfrak{D}_{N O R} \supsetneq \mathfrak{D}_{M}$. 
Chung (2000) discussed ten different no odd rings preference domains based on preference domains introduced and analyzed in the context of social choice theory by Inada (1964, 1969) and Sen and Pattanaik (1969) 9 the most well-known domains being the domain of single-peaked preferences and the domain of single-caved preferences. Another no odd rings preference domain is obtained by applying Becker's (1973) idea of production between couples (using complete rigidity in the division of output) to any pair of agents; Chung (2000) refers to this domain of markets as Beckerian unisexual marriage markets. The last of Chung's (2000) no odd rings preference domain of interest is based on Bartholdi and Trick's (1986) restriction on the preferences such that agents traits can be represented by points in a metric space, and every agent strictly prefers agents who have traits similar to his to those who have more different traits, and strictly prefers having a roommate to not. All the market domains introduced by Chung (2000) are subdomains of the domain of solvable markets without 3-rings. However, most of the market domains introduced by Chung (2000) do not contain any rings (also not even rings) and therefore they do not contain the important domain of marriage markets. The domain of solvable markets without 3-rings, for which we will obtain one of our main results (Theorem 1), does contain marriage markets.

Another well-known concept for matching markets is the core.

Definition (The Core). A matching is in the core if no coalition of agents can improve their welfare by rematching among themselves. For market $(N, R) \in \mathfrak{D}, \operatorname{core}(N, R)=\{\mu \in$ $\mathcal{M}(N) \mid$ there exists no $S \subseteq N$ and no $\mu^{\prime} \in \mathcal{M}(N)$ such that $\mu^{\prime}(S)=S$, for all $i \in S, \mu^{\prime}(i) R_{i}$ $\mu(i)$, and for some $\left.j \in S, \mu^{\prime}(j) P_{j} \mu(j)\right\}$.

Similarly as in other matching models (e.g., classical marriage markets and college admissions markets with responsive preferences), the core equals the set of stable matchings, i.e., for all $(N, R) \in \mathfrak{D}_{S}$, core $(N, R)=S(N, R)$. The core is a solution on the domain of solvable markets $\mathfrak{D}_{S}$ and all its subdomains (particularly, $\mathfrak{D}_{S N 3 R}, \mathfrak{D}_{N O R}$, and $\mathfrak{D}_{M}$ ) but not on the domain of all markets $\mathfrak{D}$.

\section{Consistency and Converse Consistency}

Consistency and converse consistency are key properties in many frameworks with variable sets of agents. Thomson (2015) provided an extensive survey of consistency and its converse for various economic models, including marriage markets. Consistency essentially requires that when a set of matched agents leaves, then the solution should still match the remaining agents as before.

Definition (Consistency). A solution $\varphi$ on $\mathfrak{D}$ is consistent if the following holds for each $(N, R) \in$ $\mathfrak{D}$ and each $\mu \in \varphi(N, R)$. If $\left(N^{\prime}, R_{N^{\prime}}\right) \in \mathfrak{D}$ is a reduced market of $(N, R)$ at $\mu$ to $N^{\prime}$ (i.e., $N^{\prime} \subseteq N$ and $\left.\mu\left(N^{\prime}\right)=N^{\prime}\right)$, then, $\mu_{N^{\prime}} \in \varphi\left(N^{\prime}, R_{N^{\prime}}\right)$.

Can and Klaus (2013, Proposition 2) show that on the domain of solvable markets and on any of its subdomains, the core satisfies consistency. The following paragraph and remark are direct adaptations from Can and Klaus (2013).

For solutions defined on $\mathfrak{D}^{\prime} \subseteq \mathfrak{D}$, consistency only applies to reduced markets $\left(N^{\prime}, R_{N^{\prime}}\right) \in \mathfrak{D}^{\prime}$. Of the five domains $\left(\mathfrak{D}_{M}, \mathfrak{D}_{N O R}, \mathfrak{D}_{S N 3 R}, \mathfrak{D}_{S}, \mathfrak{D}\right)$ that we consider, only four are closed with respect

\footnotetext{
${ }^{9}$ On each of these ten preference domains, the simple majority decision rule is guaranteed to be transitive.
} 
to the reduction operator, i.e., for $\mathfrak{D}^{\prime} \in\left\{\mathfrak{D}_{M}, \mathfrak{D}_{N O R}, \mathfrak{D}_{S N 3 R}, \mathfrak{D}\right\}$, if $(N, R) \in \mathfrak{D}^{\prime}, \mu \in \mathcal{M}(N)$, and $\left(N^{\prime}, R_{N^{\prime}}\right)$ is a reduced market of $(N, R)$ at $\mu$, then $\left(N^{\prime}, R_{N^{\prime}}\right) \in \mathfrak{D}^{\prime}$. For the domain of solvable markets $\mathfrak{D}_{S}$, non-solvable reduced markets exist and therefore consistency "loses some of its bite" (because it makes no predictions whenever market reduction leads to unsolvable reduced markets).

Remark 1 (Solvability when Studying the Core and Domain Restrictions). Since stable matchings need not exist for the general domain of all markets, we have to restrict attention to subdomains of solvable markets when studying the core. Considering the whole domain of solvable markets when studying consistency is difficult because a solvable market might well have unsolvable reduced markets. Requiring that a solution only selects matchings that guarantee the solvability of all restricted markets, would already steer results forcefully towards the core. However, three domains of markets we consider, $\mathfrak{D}_{M}, \mathfrak{D}_{N O R}$, and $\mathfrak{D}_{S N 3 R}$, satisfy "closedness and solvability under the reduction operation", i.e., for any market in $\mathfrak{D}^{\prime} \in\left\{\mathfrak{D}_{M}, \mathfrak{D}_{N O R}, \mathfrak{D}_{S N 3 R}\right\}$, all possible reduced markets are (i) elements of the domain $\mathfrak{D}^{\prime}$ and (ii) solvable; note that $\mathfrak{D}_{S N 3 R}$ is the largest domain that is closed and guarantees solvability under the reduction operation.

Lemma 1. On the domain of solvable markets without 3-rings, no proper subsolution of the core satisfies consistency.

Lemma 1 is a counterpart of a result for classical marriage markets that was first established by Toda (2006, Lemma 3.6) and then extended by Can and Klaus $(2013$, Lemma 1$)$ to the domains $\mathfrak{D}_{S}, \mathfrak{D}_{N O R}$, and $\mathfrak{D}_{M}$. The proof of Lemma 1 is the same as that of Can and Klaus (2013, Lemma 1) for $\mathfrak{D}_{N O R}$ : neither in the proof of the so-called Bracing lemma that is used nor in the proof of Can and Klaus (2013, Lemma 1) itself does it matter if markets are from $\mathfrak{D}_{S N 3 R}$ or the smaller domain $\mathfrak{D}_{N O R}$. On the domain of all markets, there is no solution that is a subsolution of the core for solvable markets and satisfies consistency (Özkal-Sanver, 2010, Proposition 4.3).

The last property we introduce is converse consistency, a property that determines the desirability of a matching for a market on the basis of the desirability of its restrictions to reduced markets that are obtained by taking two agents and their matches. So, given a matching $\mu$ for a market $(N, R)$, if for any set of two agents $N^{\prime} \subseteq N,\left|N^{\prime}\right|=2$, the restriction of $\mu$ to the set of agents $M^{\prime}:=N^{\prime} \cup \mu\left(N^{\prime}\right)$ equals the matching chosen by the solution, then $\mu$ must be a matching assigned by the solution.

Definition (Converse Consistency). A solution $\varphi$ on $\mathfrak{D}$ is conversely consistent if the following holds for each $(N, R) \in \mathfrak{D}$ and each $\mu \in \mathcal{M}(N)$. If for all $N^{\prime} \subseteq N,\left|N^{\prime}\right|=2$, and setting $M^{\prime}:=N^{\prime} \cup \mu\left(N^{\prime}\right)$, for the reduced market $\left(M^{\prime}, R_{M^{\prime}}\right) \in \mathfrak{D}$ we have $\mu_{M^{\prime}} \in \varphi\left(M^{\prime}, R_{M^{\prime}}\right)$, then $\mu \in \varphi(N, R)$.

Proposition 1. On the domain of solvable markets without 3-rings, the core satisfies individual rationality, anonymity, Pareto optimality, consistency, and converse consistency.

Proof. It is easy to see that, on any market domain, the core satisfies individual rationality, anonymity, and Pareto optimality.

Assume that the core is not consistent on domain $\mathfrak{D}_{S N 3 R}$. Then, there exist $(N, R) \in \mathfrak{D}_{S N 3 R}$, $\mu \in \operatorname{core}(N, R)$, and $N^{\prime} \subseteq N$ such that $\left(N^{\prime}, R_{N^{\prime}}\right)$ is a reduced market of $(N, R)$ at $\mu$ to $N^{\prime}$, $\left(N^{\prime}, R_{N^{\prime}}\right) \in \mathfrak{D}_{S N 3 R}$, and $\mu_{N^{\prime}} \notin \operatorname{core}\left(N^{\prime}, R_{N^{\prime}}\right)$. Hence, there exists a blocking pair $\{i, j\} \subseteq N^{\prime}$ for $\mu_{N^{\prime}}$, i.e., $j P_{i} \mu_{N^{\prime}}(i)$ and $i P_{j} \mu_{N^{\prime}}(j)$. However, since $\mu_{N^{\prime}}(i)=\mu(i)$ and $\mu_{N^{\prime}}(j)=\mu(j),\{i, j\} \subseteq N$ is also a blocking pair for $\mu$; contradicting $\mu \in \operatorname{core}(N, R)$. 
Assume that the core is not conversely consistent on domain $\mathfrak{D}_{S N 3 R}$. Then, there exist $(N, R) \in$ $\mathfrak{D}_{S N 3 R}$ and $\mu \in \mathcal{M}(N)$ such that for all $N^{\prime} \subseteq N,\left|N^{\prime}\right|=2$, setting $M^{\prime}:=N^{\prime} \cup \mu\left(N^{\prime}\right)$, (i) $\left(M^{\prime}, R_{M^{\prime}}\right) \in \mathfrak{D}_{S N 3 R}$, (ii) $\mu_{M^{\prime}} \in \operatorname{core}\left(M^{\prime}, R_{M^{\prime}}\right)$, and (iii) $\mu \notin \operatorname{core}(N, R){ }^{10}$ Hence, there exists a blocking pair $\{i, j\} \subseteq N$ for $\mu$. Let $\bar{N}^{\prime}=\{i, j\}$ and $\bar{M}^{\prime}:=\bar{N}^{\prime} \cup \mu\left(\bar{N}^{\prime}\right)$. Then, $\{i, j\}$ is also a blocking pair for $\mu_{\bar{M}^{\prime}}$; a contradiction.

Note that Proposition 1 can similarly be established on preference domains $\mathfrak{D}_{M}$ and $\mathfrak{D}_{N O R}$. On the domain of solvable markets $\mathfrak{D}_{S}$, the core is consistent (by the same arguments as in the proof of consistency in Proposition 1) but it is not conversely consistent.

Proposition 2. On the domain of solvable markets, the core does not satisfy converse consistency.

Proof. Let market $(\hat{N}, \hat{R}) \in \mathfrak{D}_{S}$ be such that $\hat{N}=\{1,2,3,4,5,6\}$ and

$$
\begin{aligned}
& \begin{array}{l|lll}
\hat{R}_{1} & 233 \text { (1) ... } & \mu=\{(1,3),(2,6),(4,5)\}
\end{array} \\
& \hat{R}_{2} \text { (3) } 612 \ldots \quad \hat{\mu}=\{(2,3),(4,6), 1,5\} \\
& \hat{R}_{3} 123 \ldots \\
& \hat{R}_{4} 564 \ldots \quad \operatorname{core}(\hat{N}, \hat{R})=\{\mu\} \\
& \hat{R}_{5} 64 \text { (5)... } \\
& \hat{R}_{6} \text { (4) } 256 \ldots
\end{aligned}
$$

We only list the individually rational part of agents' preferences and we indicate matching $\hat{\mu}=$ $\{(2,3),(4,6), 1,5\}$ above by a circle around an agent's match. Market $(\hat{N}, \hat{R})$ is solvable and contains two 3 -rings $\{1,2,3\}$ and $\{4,5,6\}$. Matching $\hat{\mu} \in(P O(\hat{N}, \hat{R}) \cap I R(\hat{N}, \hat{R})) \backslash \operatorname{core}(\hat{N}, \hat{R})$ (since $\{1,3\}$ blocks $\hat{\mu} ;\{4,5\}$ also does). We assume, by contradiction, that the core is conversely consistent.

Consider $i, j \in \hat{N}$ and set $M^{\prime}:=\{i, j, \hat{\mu}(i), \hat{\mu}(j)\}$. Note that the reduced markets $\left(M^{\prime}, \hat{R}_{M^{\prime}}\right)$ that contain a blocking pair, i.e., $M^{\prime} \in\{\{1,2,3\},\{4,5,6\}\}$, are not solvable and thus converse consistency does not require anything. For all remaining reduced market $\left(M^{\prime}, \hat{R}_{M^{\prime}}\right), \hat{\mu}_{M^{\prime}}$ is a stable matching. Hence, for all $N^{\prime} \subseteq \hat{N},\left|N^{\prime}\right|=2$, setting $M^{\prime}:=N^{\prime} \cup \hat{\mu}\left(N^{\prime}\right)$, and the reduced market $\left(M^{\prime}, R_{M^{\prime}}\right) \in \mathfrak{D}_{S}, \hat{\mu}_{M^{\prime}} \in \operatorname{core}\left(M^{\prime}, R_{M^{\prime}}\right)$. Then, by converse consistency, $\hat{\mu} \in \operatorname{core}(\hat{N}, \hat{R})$; a contradiction.

\section{Characterizing the Core}

\subsection{Previous Results for Classical Marriage Markets}

For a subdomain of the domain of classical marriage markets for which matching agents of the same gender is not feasible, Sasaki and Toda (1992) characterized the core by same-side anonymity for marriage markets, Pareto optimality, consistency, and converse consistency. Sasaki and Toda (1992) used the term anonymity for same-side anonymity for marriage markets to require that matchings assigned by the solution do not depend on agents' names when the men's names are permuted on the men's side of the market or when the women's names are permuted on the women's side of the market 11

\footnotetext{
${ }^{10}$ Note that this statement uses the fact that on domain $\mathfrak{D}_{S N 3 R}$ all reduced markets are again in $\mathfrak{D}_{S N 3 R}$.

${ }^{11}$ Formally, a solution $\varphi$ is same-side anonymous for marriage markets if for all marriage markets $(M \cup W, R)$ and all permutations $\pi$ such that $M^{\pi}=M$ and $W^{\pi}=W, \mu \in \varphi(M \cup W, R)$ implies $\mu^{\pi} \in \varphi\left(M^{\pi} \cup W^{\pi}, R^{\pi}\right)$.
} 
Theorem A (Sasaki and Toda (1992), Main Theorem). On the domain of classical marriage markets with equal numbers of men and women and where all men find all women acceptable and all women find all men acceptable, a solution satisfies same-side anonymity for marriage markets, Pareto optimality, consistency, and converse consistency if and only if it is the core.

Nizamogullari and Özkal-Sanver (2014) generalized this result to the domain of classical marriage markets by adding individual rationality and replacing same-side anonymity for marriage markets with a stronger property called gender fairness (introduced in Özkal-Sanver, 2004). Gender fairness requires that for marriage markets with an equal number of men and women, renaming men as women, and renaming women as men, does not change the outcomes chosen by the solution when taking the renaming of agents into account ${ }^{12}$ We will discuss their characterization result in view of ours in Section 4.2 (after the proof of Theorem 1).

Theorem B (Nizamogullari and Özkal-Sanver (2014), Theorem 3.1). On the domain of classical marriage markets, a solution satisfies individual rationality, Pareto optimality, consistency, converse consistency, and gender fairness if and only if it is the core.

Özkal-Sanver (2010, Proposition 4.2) showed that on the domain of all markets, no solution satisfies Pareto optimality, anonymity, and converse consistency. However, the proof of this impossibility result used an unsolvable market. Hence, the question whether or not the characterization of Sasaki and Toda (1992) can be extended to the domain of solvable markets or to any of its subdomains has not been answered until now.

In the next section, we will first extend the characterizations of Sasaki and Toda $(1992)$ and Nizamogullari and Özkal-Sanver (2014) to the domain of solvable markets without 3-rings (Theorem 1). Second, we will show that the same properties characterize the core for marriage markets (Theorem 2). Third, we demonstrate that the properties of Theorem 1 are compatible on the domain of solvable roommate markets by constructing a conversely consistent extension of the core (Proposition 3).

\subsection{Core Characterizations for Roommate and Marriage Markets}

First, we prove that the characterizations of Sasaki and Toda (1992) and Nizamogullari and ÖzkalSanver (2014) for classical marriage markets can be extended to roommate markets for the domain of solvable markets without 3-rings.

Theorem 1 (A Characterization of the Core). On the domain of solvable markets without 3-rings, a solution satisfies individual rationality, anonymity, Pareto optimality, consistency, and converse consistency if and only if it is the core.

Remark 2 (An Alternative Characterization of the Core). A solution is individually/mutually best if an agent who finds being single best will always be single and if two "mutually best agents" are always matched with each other.

Definition (Individually/Mutually Best). A solution $\varphi$ on $\mathfrak{D}$ is individually/mutually best if the following holds for each market $(N, R): i, j \in N$ [possibly $i=j$ ] such that for all $k \in N, i R_{j} k$ and $j R_{i} k$ implies that for all $\mu \in \varphi(N, R), \mu(i)=j$.

\footnotetext{
${ }^{12}$ Formally, a solution $\varphi$ is gender fair if for all marriage markets $(M \cup W, R)$ such that $|M|=|W|$ and all permutations $\pi$ such that $M^{\pi}=W$ and $W^{\pi}=M, \mu \in \varphi(M \cup W, R)$ implies $\mu^{\pi} \in \varphi\left(M^{\pi} \cup W^{\pi}, R^{\pi}\right)$.
} 
Can and Klaus (2013, Lemma 2) show on various preference domains that individually/mutually best and consistency imply individual rationality. The proof of this lemma does not depend on the preference domain and hence the lemma also holds on the domain of solvable markets without 3rings. Thus, we obtain an alternative characterization of the core by replacing individual rationality with individually/mutually best in Theorem 1 .

Proof of Theorem 1. By Proposition 1, the core satisfies all the properties of the theorem on $\mathfrak{D}_{S N 3 R}$.

To prove the uniqueness part, let $\varphi$ be a solution on $\mathfrak{D}_{S N 3 R}$ that satisfies individual rationality, anonymity, Pareto optimality, consistency, and converse consistency. We now proceed in two steps. First, in Part I of the uniqueness proof, we show that solution $\varphi$ will never choose unstable matchings, i.e., for all $(N, R) \in \mathfrak{D}_{S N 3 R}, \varphi(N, R) \subseteq \operatorname{core}(N, R)$. Second, in Part II of the uniqueness proof, we use Lemma 1 to conclude that for all $(N, R) \in \mathfrak{D}_{S N 3 R}, \varphi(N, R)=\operatorname{core}(N, R)$.

\section{Uniqueness Part I: $\varphi \subseteq$ core}

We prove $\varphi \subseteq$ core by contradiction. Assume that for some solvable market without a 3-ring an unstable and individually rational matching is chosen. Then, by consistency, in the reduced market that contains a blocking pair and their matches, the solution chooses the corresponding unstable and individually rational reduced matching. If the reduced market is of size 2 , a contradiction to Pareto optimality is immediately obtained. If the reduced market is of size 3 or 4, using anonymity, we combine several reduced markets that we derive from the original reduced market into a larger solvable market without 3-rings. By anonymity, we know that a specific (unstable and individually rational) matching for each reduced market has to be chosen and, by converse consistency, this results in a matching being chosen for the larger market that is not Pareto optimal. We again obtain a contradiction. We prove $\varphi \subseteq$ core in Appendix A.

\section{Uniqueness Part II: $\varphi=$ core}

We have proven that $\varphi \subseteq$ core. By Lemma 1, no proper subsolution of the core satisfies consistency. Hence, $\varphi=$ core.

We prove the independence of properties of Theorem 1 in Appendix $B$.

Next, consider the marriage market domain $\mathfrak{D}_{M}$. Recall that we model the classical marriage market restriction that no two men and no two women are matched via the agents' preferences together with individual rationality: no woman finds another woman acceptable and no man finds another man acceptable. Our anonymity property, for any marriage market, will respect the partition into men and women that is embedded in the agents' preferences. Now, the proof of our characterization (Theorem 1) can easily be adapted to show the following marriage market characterization (see Appendix C).

Theorem 2 (A Characterization of the Core for Marriage Markets). On the domain of marriage markets, a solution satisfies individual rationality, anonymity, Pareto optimality, consistency, and converse consistency if and only if it is the core.

Nizamogullari and Özkal-Sanver (2014, Example 3.2) showed for classical marriage markets that the corresponding characterization by Sasaki and Toda (1992), which was established for a smaller domain, does not hold anymore. The reason why, on the domain of classical marriage markets, a different solution from the core satisfies the properties individual rationality, same-side anonymity for marriage markets, Pareto optimality, consistency, and converse consistency is that 
same-side anonymity for marriage markets only allows to rename men within the group of men and women within the group of women. Hence, same-side anonymity for marriage markets as introduced by Sasaki and Toda (1992) and adapted by Nizamogullari and Özkal-Sanver (2014) still allows a solution to discriminate based on gender and hence does not capture the full spirit of anonymity. It suffices to strengthen same-side anonymity for marriage markets by adding the requirement that whenever there is an equal number of men and women present, renaming men as women and women as men does not essentially change the matchings a solution assigns (up to the corresponding name changes): this stronger property is referred to as gender fairness (Özkal-Sanver, 2004: Nizamogullari and Özkal-Sanver, 2014).

Özkal-Sanver (2010, Proposition 4.2) showed that on the domain of all markets, no solution satisfies Pareto optimality, anonymity, and converse consistency. By Proposition 2, the core does not satisfy converse consistency on the domain of solvable markets. However, the properties in Theorem 1 are compatible on the domain of solvable markets. We show this by using the insights that we have gained from the proof of Proposition 2 and by adding unstable matchings to the core to obtain a solution that will satisfy converse consistency.

Proposition 3. On the domain of solvable markets, there exists a solution $\hat{\varphi} \supsetneq$ core that satisfies individual rationality, anonymity, Pareto optimality, consistency, and converse consistency.

Proof. For any solvable market $(N, R) \in \mathfrak{D}_{S}$, we first define the set of "added matchings" $A(N, R)$ by $\mu \in A(N, R)$ if and only if $\mu \in(P O(N, R) \cap I R(N, R)) \backslash \operatorname{core}(N, R)$ and for all $N^{\prime} \subseteq N,\left|N^{\prime}\right|=2$, setting $M^{\prime}:=N^{\prime} \cup \mu\left(N^{\prime}\right)$, for the reduced market $\left(M^{\prime}, R_{M^{\prime}}\right) \in \mathfrak{D}_{S}$ we have $\mu_{M^{\prime}} \in \operatorname{core}\left(M^{\prime}, R_{M^{\prime}}\right)$. Then, for all solvable markets $(N, R) \in \mathfrak{D}_{S}$,

$$
\hat{\varphi}(N, R):=\operatorname{core}(N, R) \cup A(N, R) .
$$

By the proof of Proposition 2 for some markets $(\hat{N}, \hat{R}) \in \mathfrak{D}_{S}, A(\hat{N}, \hat{R}) \neq \emptyset$ and hence, $\hat{\varphi} \supsetneq$ core.

Solution $\hat{\varphi}$, by construction, satisfies individual rationality, anonymity, Pareto optimality, and consistency (the core is consistent and the added matchings have the property that for all the corresponding reduced markets a core matching is chosen).

To show converse consistency, we need to show that we won't have to add any additional matchings. Assume, by contradiction, that there exist $(\tilde{N}, \tilde{R}) \in \mathfrak{D}_{S}$ and $\tilde{\mu} \notin P O(\tilde{N}, \tilde{R}) \cap I R(\tilde{N}, \tilde{R})$ such that for all $N^{\prime} \subseteq \tilde{N},\left|N^{\prime}\right|=2$, setting $M^{\prime}:=N^{\prime} \cup \mu\left(N^{\prime}\right)$, for the reduced market $\left(M^{\prime}, R_{M^{\prime}}\right) \in$ $\mathfrak{D}_{S}$ we have $\tilde{\mu}_{M^{\prime}} \in \operatorname{core}\left(M^{\prime}, R_{M^{\prime}}\right)$. First, if $\tilde{\mu} \notin I R(\tilde{N}, \tilde{R})$, then for some $i \in \tilde{N}, i P_{i} \tilde{\mu}(i)$ and for $N^{\prime}=M^{\prime}=\{i, \tilde{\mu}(i)\}, \tilde{\mu}_{M^{\prime}} \notin \operatorname{core}\left(M^{\prime}, R_{M^{\prime}}\right)$; a contradiction. Second, if $\tilde{\mu} \notin P O(\tilde{N}, \tilde{R})$, then there exists a Pareto improvement $\bar{\mu}$ of $\tilde{\mu}$ such that for all $i \in \tilde{N}, \bar{\mu}(i) R_{i} \tilde{\mu}(i)$ and for some $j \in \tilde{N}$, $\bar{\mu}(j) P_{j} \tilde{\mu}(j)$. Let $k=\bar{\mu}(j)$ and note that $k P_{j} \tilde{\mu}(j)$ and $j P_{k} \tilde{\mu}(k)$. Hence, for $N^{\prime}=\{i, k\}$ and setting $M^{\prime}:=\{i, k, \tilde{\mu}(i), \tilde{\mu}(k)\},\{j, k\}$ is a blocking pair for $\tilde{\mu}_{M^{\prime}}$ and $\tilde{\mu}_{M^{\prime}} \notin \operatorname{core}\left(M^{\prime}, R_{M^{\prime}}\right)$; a contradiction.

Remark 3 (The Domain of Solvable No-3-Ring Markets and an Open Question). We establish our core characterization for roommate markets on the domain of solvable no-3-ring markets. The reason why higher-order odd rings will never cause any problems in combination with the properties basically is that they cannot appear in reduced markets since these markets contain at most four agents. In the proof of the characterization (Theorem 1, Appendix A), starting from an unstable matching and a four agent reduced market $(N, R)$ constructed from one of its blocking pairs, we construct a larger eight agent market $(\tilde{N}, \tilde{R})$ for which we then obtain a contradiction. 
There are hundreds of possible preference combinations for market $(\tilde{N}, \tilde{R})$ and while we manage to prove that it never contains any 3 -rings given that $(N, R)$ did not contain any 3 -rings, we do not see any way with our current proof approach to show that it does not contain 5-rings or 7-rings. Whether a corresponding characterization of the core on the domain of no odd rings exists is an open question.

\section{Appendix}

\section{A Proof of Theorem 1, Uniqueness Part I: $\varphi \subseteq$ core}

Assume, by contradiction, that there exists a solvable market without 3-rings $(\bar{N}, \bar{R}) \in \mathfrak{D}_{S N 3 R}$ such that $\varphi(\bar{N}, \bar{R}) \nsubseteq \operatorname{core}(\bar{N}, \bar{R})$. Then, there exists a matching $\bar{\mu} \in \varphi(\bar{N}, \bar{R})$ with a blocking pair $\{i, j\}$ for $\bar{\mu}$. By individual rationality, $i \neq j$. Let $N^{\prime}=\{i, j\}$. Set $N=N^{\prime} \cup \bar{\mu}\left(N^{\prime}\right)=\{i, j, \bar{\mu}(i), \bar{\mu}(j)\}$, $R=\bar{R}_{N}$, and $\mu=\bar{\mu}_{N}$. Consider the reduced market $(N, R) \in \mathfrak{D}_{S N 3 R}$. By consistency, $\mu \in \varphi(N, R)$. Since $\{i, j\}$ is a blocking pair for $\mu, \mu$ is not stable (i.e., $\mu \in \varphi(N, R) \backslash \operatorname{core}(N, R)$ ). We consider three cases depending on the cardinality of $N$.

In the sequel, we indicate matching $\mu$ (or corresponding other matchings being discussed at the time) in preference tables by a circle around an agent's match.

Case $1(|N|=2)$ : Then, $N=\{i, j\}$ and $\{i, j\}$ being a blocking pair for $\mu$ implies that agents' preferences are as follows:

$$
\begin{array}{l|ll}
R_{i} & j & i \\
R_{j} & i & j
\end{array}
$$

However, note that agents $i$ and $j$ would both prefer being matched with each other to being single at $\mu$; a contradiction to Pareto optimality.

Case $2(|N|=3)$ : Without loss of generality, let $k=\mu(i)$ and $j$ is single at $\mu$. Then, $N=\{i, j, k\}$. By individual rationality and $\{i, j\}$ being a blocking pair for $\mu$, agents' partial preferences are as follows (the possible positions of agent $k$ in $R_{j}$ are denoted by ${ }_{(k)}$ and the possible positions of agent $j$ in $R_{k}$ are denoted by $(j)$ :

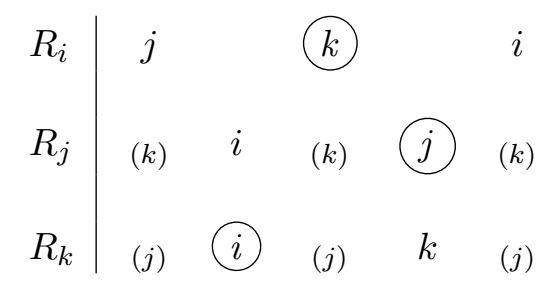

There is exactly one unsolvable market that complies with the above partial preferences (it is a market composed of a 3-ring):

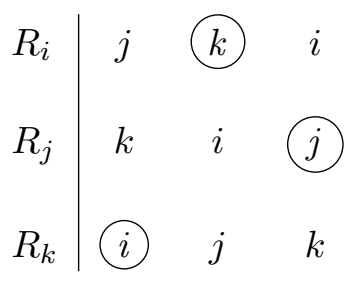


It is noteworthy to mention that the above preference profile illustrates why we need to exclude 3 -rings in our theorem. The above market is not solvable and solution $\varphi$ is not defined for this reduced market. Therefore, for this specific preference profile, we would not be able to obtain the desired contradiction. We proceed with the proof knowing that none of the reduced markets we consider contains a 3 -ring.

Preferences $R_{j}$ and $R_{k}$ above are incomplete and we can distinguish 8 cases of complete preferences $R_{j}$ and $R_{k}$ without a 3-ring. Relying heavily on anonymity, we will deal with all possible cases at the same time, but the interested reader can find a case-by-case proof in Klaus (2017, Appendix D).

Let $\pi_{a} \in \Pi^{\mathbb{N}}$ be such that

$$
\pi_{a}(i)=1, \pi_{a}(j)=3, \text { and } \pi_{a}(k)=2
$$

and let $\pi_{b} \in \Pi^{\mathbb{N}}$ be such that

$$
\pi_{b}(i)=2, \pi_{b}(j)=4, \text { and } \pi_{b}(k)=1 .
$$

For $\tilde{N}=\{1,2,3,4\}$ we define matching $\tilde{\mu}:=\{(1,2), 3,4\}$ and $\tilde{R} \in \mathcal{R}^{\tilde{N}}$ as follows:

$\tilde{R}_{1}$ is such that

(1.1) $\tilde{R}_{1} \mid \begin{array}{lll}3 & 2 & 1\end{array}$

(1.2) $\tilde{R}_{1} \mid 3 \quad 4$, and

(1.3) the relative position of 4 with respect to 2 and 1 is that induced by their respective images under $\pi_{b}$ in $R_{k}$.

$\tilde{R}_{2}$ is such that

(2.1) $\tilde{R}_{2} \mid \begin{array}{lll}4 & 1 & 2\end{array}$

(2.2) $\tilde{R}_{2} \mid 4 \quad 3$, and

(2.3) the relative position of 3 with respect to 1 and 2 is that induced by their respective images under $\pi_{a}$ in $R_{k}$.

$\tilde{R}_{3}$ is such that

(3.1) $\tilde{R}_{3} \mid \begin{array}{lll} & 3 & 4\end{array}$

(3.2) $\tilde{R}_{3} \mid 2$ 4, and

(3.3) the relative position of 2 with respect to 1 and 3 is that induced by their respective images under $\pi_{a}$ in $R_{j}$.

$\tilde{R}_{4}$ is such that

(4.1) $\tilde{R}_{4} \mid 2 \quad 4 \quad 3$,

(4.2) $\tilde{R}_{4} \mid 13$, and 
(4.3) the relative position of 1 with respect to 2 and 4 is that induced by their respective images under $\pi_{b}$ in $R_{j}$.

We summarize $\tilde{R}$ and matching $\tilde{\mu}$ by writing

\begin{tabular}{c|cccccc}
$\tilde{R}_{1}$ & 3 & $(4)$ & 2 & $(4)$ & 1 & $(4)$ \\
$\tilde{R}_{2}$ & 4 & $(3)$ & $(1)$ & $(3)$ & 2 & $(3)$ \\
$\tilde{R}_{3}$ & $(2)$ & 1 & $(2)$ & 3 & $(2)$ & 4 \\
$\tilde{R}_{4}$ & $(1)$ & 2 & $(1)$ & $(4)$ & $(1)$ & 3
\end{tabular}

To check that $(\tilde{N}, \tilde{R})$ does not contain a 3-ring we only need to show that no three agent set $N^{\prime}=\left\{i_{1}, i_{2}, i_{3}\right\} \subseteq\{1,2,3,4\}$ induces a corresponding 3-ring market $\left(N^{\prime}, \tilde{R}_{N^{\prime}}\right)$. There are $\left(\begin{array}{l}4 \\ 3\end{array}\right)=4$ such sets: $\{1,2,3\},\{1,2,4\},\{1,3,4\}$, and $\{2,3,4\}$.

For $N^{\prime}=\{1,2,3\}$ or $N^{\prime}=\{1,2,4\}$ this follows by anonymity because $\left(N^{\prime}, \tilde{R}_{N^{\prime}}\right)$ is a reduced market of $\left(N^{\pi_{a}}, R^{\pi_{a}}\right)$ or $\left(N^{\pi_{b}}, R^{\pi_{b}}\right)$ and the original market $(N, R)$ does not have a 3 -ring.

Since $3 \tilde{R}_{3} 4$ and $4 \tilde{R}_{4} 3$, agents 3 and 4 will never be in a 3 -ring together and hence neither $N^{\prime}=\{1,3,4\}$ nor $N^{\prime}=\{2,3,4\}$ induce 3-ring markets.

Next, we show that for all $N^{\prime} \subseteq \tilde{N},\left|N^{\prime}\right|=2$, setting $M^{\prime}:=N^{\prime} \cup \tilde{\mu}\left(N^{\prime}\right)$, and the reduced market $\left(M^{\prime}, \tilde{R}_{M^{\prime}}\right), \tilde{\mu}_{M^{\prime}} \in \varphi\left(M^{\prime}, \tilde{R}_{M^{\prime}}\right)$. Note that $\left(M^{\prime}, \tilde{R}_{M^{\prime}}\right) \in \mathfrak{D}_{S N 3 R}$. There are $\left(\begin{array}{l}4 \\ 2\end{array}\right)=6$ sets $N^{\prime}$ to check.

1. $N^{\prime}=M^{\prime}=\{1,2\}$ :

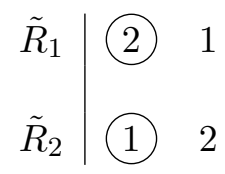

Pareto optimality implies that $\tilde{\mu}_{M^{\prime}} \in \varphi\left(M^{\prime}, \tilde{R}_{M^{\prime}}\right)$.

2. $N^{\prime}=M^{\prime}=\{3,4\}$ :

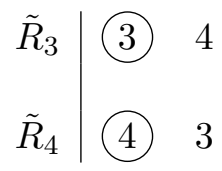

Pareto optimality implies that $\tilde{\mu}_{M^{\prime}} \in \varphi\left(M^{\prime}, \tilde{R}_{M^{\prime}}\right)$.

3. \& 4. $N^{\prime}=\{1,3\}$ or $N^{\prime}=\{2,3\}$ and $M^{\prime}=\{1,2,3\}$ :
$\tilde{R}_{1} \mid 3$
(2)
$1 \stackrel{\pi_{a}}{\longleftarrow} R_{i}$
(k)
$i$
$\tilde{R}_{3} \quad(2) \quad 1$
(2)
(3)
(2) $\quad \stackrel{\pi_{a}}{\longleftarrow} \quad R_{j} \quad(k) \quad i$
(k) j
\begin{tabular}{l|l}
$\tilde{R}_{2}$ & (3)
\end{tabular}
(3) 2
(3)
$\stackrel{\pi_{a}}{\longleftarrow} \quad R_{k} \quad{ }_{(j)}$
(i)
(j) $k$
$(j)$ 
Note that $N^{\pi_{a}}=M^{\prime}$. In the above preference table, we have already indicated that $\tilde{R}_{M^{\prime}}=$ $R^{\pi_{a}}$. Then, by anonymity, $\tilde{\mu}_{M^{\prime}}=\mu^{\pi_{a}} \in \varphi\left(M^{\prime}, \tilde{R}_{M^{\prime}}\right)$.

5. \& 6. $N^{\prime}=\{1,4\}$ or $N^{\prime}=\{2,4\}$ and $M^{\prime}=\{1,2,4\}$ :

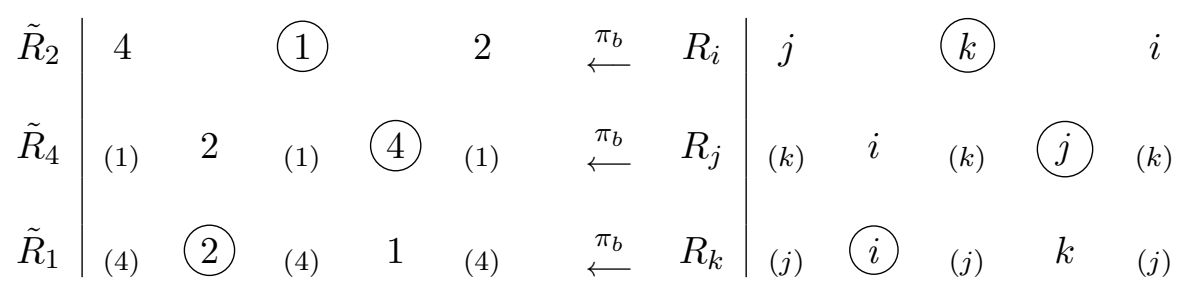

Note that $N^{\pi_{b}}=M^{\prime}$. In the above preference table, we have already indicated that $\tilde{R}_{M^{\prime}}=$ $R^{\pi_{b}}$. Then, by anonymity, $\tilde{\mu}_{M^{\prime}}=\mu^{\pi_{b}} \in \varphi\left(M^{\prime}, \tilde{R}_{M^{\prime}}\right)$.

We have shown that for all $N^{\prime} \subseteq \tilde{N},\left|N^{\prime}\right|=2$, setting $M^{\prime}:=N^{\prime} \cup \tilde{\mu}\left(N^{\prime}\right)$, and $\left(M^{\prime}, \tilde{R}_{M^{\prime}}\right) \in$ $\mathfrak{D}_{S N 3 R}, \tilde{\mu}_{M^{\prime}} \in \varphi\left(M^{\prime}, \tilde{R}_{M^{\prime}}\right)$. Hence, by converse consistency, $\tilde{\mu} \in \varphi(\tilde{N}, \tilde{R})$. However, note that matching $\{(1,3),(2,4)\}$ is strictly preferred by everybody; a contradiction to Pareto optimality.

Case $3(|N|=4)$ : Let $k=\mu(i), l=\mu(j)$, and $N=\{i, j, k, l\}$. By individual rationality and $\{i, j\}$ being a blocking pair for $\mu$, agents' partial preferences are as follows (the possible positions of agent $l$ in $R_{i}$ are denoted by ${ }_{(l)}$, the possible positions of agent $k$ in $R_{j}$ are denoted by ${ }_{(k)}$, the possible positions of agents $j$ and $l$ in $R_{k}$ are denoted by ${ }_{(j),(l)}$, and the possible positions of agents $i$ and $k$ in $R_{l}$ are denoted by $\left.{ }_{(i),(k)}\right)$ :

\begin{tabular}{|c|c|c|c|c|c|c|}
\hline$R_{i}$ & $(l)$ & $j$ & $(l)$ & (k) & $(l)$ & $i$ \\
\hline$R_{j}$ & $(k)$ & $i$ & $(k)$ & (l) & $(k)$ & $j$ \\
\hline$R_{k}$ & & $(j),(l)$ & $i$ & $(j),(l)$ & $k$ & $(j),(l)$ \\
\hline$R_{l}$ & & $(i),(k)$ & & $(i),(k)$ & $l$ & $(i),(k)$ \\
\hline
\end{tabular}

There are $4 \cdot 4 \cdot 12 \cdot 12=2304$ preference profiles that agree with the above partial preferences. Even though some of these profiles are not part of the solvable domain without 3-rings and for some of these profiles $\mu$ is not Pareto optimal, a case-by-case proof (as for Case 2 in Klaus, 2017, Appendix D), is a bit too much work. Luckily, as in Case 2, we can offer a compact proof using anonymity. From now on we assume that the above market $(N, R)$ is a solvable market without 3 -rings and that matching $\mu$ is Pareto optimal.

Let $\pi_{a} \in \Pi^{\mathbb{N}}$ be such that

$$
\pi_{a}(i)=1, \pi_{a}(j)=3, \pi_{a}(k)=2, \text { and } \pi_{a}(l)=4,
$$

let $\pi_{b} \in \Pi^{\mathbb{N}}$ be such that

$$
\pi_{b}(i)=2, \pi_{b}(j)=6, \pi_{b}(k)=1 \text { and } \pi_{b}(l)=5,
$$


let $\pi_{c} \in \Pi^{\mathbb{N}}$ be such that

$$
\pi_{c}(i)=7, \pi_{c}(j)=5, \pi_{c}(k)=8 \text { and } \pi_{c}(l)=6,
$$

and let $\pi_{d} \in \Pi^{\mathbb{N}}$ be such that

$$
\pi_{d}(i)=8, \pi_{d}(j)=4, \pi_{d}(k)=7 \text { and } \pi_{d}(l)=3 .
$$

For $\tilde{N}=\{1,2,3,4,5,6,7,8\}$ we define matching $\tilde{\mu}:=\{(1,2),(3,4),(5,6),(7,8)\}$ and $\tilde{R} \in \mathcal{R}^{\tilde{N}}$ as follows:

$\tilde{R}_{1}$ is such that

(1.1) $\tilde{R}_{1} \mid \begin{array}{lllll}3 & 2 & 1 & 7 & 8\end{array}$

(1.2) $\tilde{R}_{1} \mid \begin{array}{lllllllll}3 & 6 & 7 & 8 & \text { and } \tilde{R}_{1} \mid & 3 & 5 & 7 & 8\end{array}$

(1.3) the relative positions of 6 and 5 with respect to 2 and 1 is that induced by their respective images under $\pi_{b}$ in $R_{k}$,

(1.4) $\tilde{R}_{1} \mid \begin{array}{lll}4 & 7 & 8 \text {, and }\end{array}$

(1.5) the relative position of 4 with respect to 3,2 , and 1 is that induced by their respective images under $\pi_{a}$ in $R_{i}$.

$\tilde{R}_{2}$ is such that

(2.1) $\tilde{R}_{2} \mid \begin{array}{lllll}6 & 1 & 2 & 7 & 8,\end{array}$

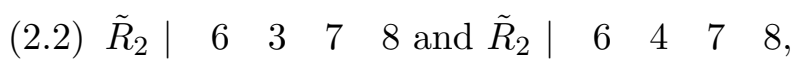

(2.3) the relative positions of 3 and 4 with respect to 1 and 2 is that induced by their respective images under $\pi_{a}$ in $R_{k}$,

(2.4) $\tilde{R}_{2} \mid \begin{array}{lll}5 & 7 & 8 \text {, and }\end{array}$

(2.5) the relative position of 5 with respect to 6,1 , and 2 is that induced by their respective images under $\pi_{b}$ in $R_{i}$.

$\tilde{R}_{3}$ is such that

(3.1) $\tilde{R}_{3} \mid \begin{array}{lllll}1 & 4 & 3 & 5 & 6\end{array}$

(3.2) $\tilde{R}_{3} \mid \begin{array}{llllllllll}1 & 8 & 5 & 6 & \text { and } \tilde{R}_{3} \mid & 1 & 7 & 5 & 6,\end{array}$

(3.3) the relative positions of 8 and 7 with respect to 4 and 3 is that induced by their respective images under $\pi_{d}$ in $R_{l}$,

(3.4) $\tilde{R}_{3} \mid \begin{array}{lll}2 & 5 & 6\end{array}$, and

(3.5) the relative position of 2 with respect to 1,4 , and 3 is that induced by their respective images under $\pi_{a}$ in $R_{j}$.

$\tilde{R}_{4}$ is such that 


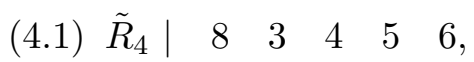

(4.2) $\tilde{R}_{4} \mid \begin{array}{lllllllll}8 & 1 & 5 & 6 & 6 \text { and } \tilde{R}_{4} \mid & 8 & 2 & 5 & 6\end{array}$

(4.3) the relative positions of 1 and 2 with respect to 3 and 4 is that induced by their respective images under $\pi_{a}$ in $R_{l}$,

(4.4) $\tilde{R}_{4} \mid \begin{array}{lll}7 & 5 & 6\end{array}$, and

(4.5) the relative position of 7 with respect to 8,3 , and 4 is that induced by their respective images under $\pi_{d}$ in $R_{j}$.

$\tilde{R}_{5}$ is such that

(5.1) $\tilde{R}_{5} \mid \begin{array}{lllll}7 & 6 & 5 & 3 & 4,\end{array}$

(5.2) $\quad \tilde{R}_{5} \mid \begin{array}{llllllllll}7 & 2 & 3 & 4 \text { and } \tilde{R}_{5} \mid & 7 & 1 & 3 & 4,\end{array}$

(5.3) the relative positions of 2 and 1 with respect to 6 and 5 is that induced by their respective images under $\pi_{b}$ in $R_{l}$,

(5.4) $\tilde{R}_{5} \mid \begin{array}{llll}8 & 3 & 4 & 4\end{array}$, and

(5.5) the relative position of 8 with respect to 7,6 , and 5 is that induced by their respective images under $\pi_{c}$ in $R_{j}$.

$\tilde{R}_{6}$ is such that

(6.1) $\tilde{R}_{6} \mid \begin{array}{lllll}2 & 5 & 6 & 3 & 4,\end{array}$

(6.2) $\tilde{R}_{6} \mid \begin{array}{lllllllll}2 & 7 & 3 & 4 \text { and } \tilde{R}_{6} \mid & 2 & 8 & 3 & 4,\end{array}$

(6.3) the relative positions of 7 and 8 with respect to 5 and 6 is that induced by their respective images under $\pi_{c}$ in $R_{l}$,

(6.4) $\tilde{R}_{6} \mid \begin{array}{lll}1 & 3 & 4\end{array}$, and

(6.5) the relative position of 1 with respect to 2,5 , and 6 is that induced by their respective images under $\pi_{b}$ in $R_{j}$.

$\tilde{R}_{7}$ is such that

(7.1) $\tilde{R}_{7} \mid \begin{array}{llllll}5 & 8 & 7 & 1 & 2,\end{array}$

$(7.2) \tilde{R}_{7} \mid \begin{array}{llllllllll}5 & 4 & 1 & 2 & \text { and } \tilde{R}_{7} & 5 & 3 & 1 & 2,\end{array}$

(7.3) the relative positions of 4 and 3 with respect to 8 and 7 is that induced by their respective images under $\pi_{d}$ in $R_{k}$,

(7.4) $\tilde{R}_{7} \mid \begin{array}{lll}6 & 1 & 2 \text {, and }\end{array}$

(7.5) the relative position of 6 with respect to 5,8 , and 7 is that induced by their respective images under $\pi_{c}$ in $R_{i}$. 
$\tilde{R}_{8}$ is such that

(8.1) $\tilde{R}_{8} \mid \begin{array}{lllll}4 & 7 & 8 & 1 & 2,\end{array}$

(8.2) $\tilde{R}_{8} \mid \begin{array}{lllllllll}4 & 5 & 1 & 2 & \text { and } \tilde{R}_{8} \mid & 4 & 6 & 1 & 2,\end{array}$

(8.3) the relative positions of 5 and 6 with respect to 7 and 8 is that induced by their respective images under $\pi_{c}$ in $R_{k}$,

(8.4) $\tilde{R}_{8} \mid \begin{array}{lll}3 & 1 & 2 \text {, and }\end{array}$

(8.5) the relative position of 3 with respect to 4,7 , and 8 is that induced by their respective images under $\pi_{d}$ in $R_{i}$.

We summarize $\tilde{R}$ and matching $\tilde{\mu}$ by writing

\begin{tabular}{l|ccccccccc}
$\tilde{R}_{1}$ & $(4)$ & 3 & $(4),(6),(5)$ & 2 & $(4),(6),(5)$ & 1 & $(4),(6),(5)$ & 7 & 8 \\
$\tilde{R}_{2}$ & $(5)$ & 6 & $(5),(3),(4)$ & 1 & $(5),(3),(4)$ & 2 & $(5),(3),(4)$ & 7 & 8 \\
$\tilde{R}_{3}$ & $(2)$ & 1 & $(2),(8),(7)$ & 4 & $(2),(8),(7)$ & 3 & $(2),(8),(7)$ & 5 & 6 \\
$\tilde{R}_{4}$ & $(7)$ & 8 & $(7),(1),(2)$ & 3 & $(7),(1),(2)$ & 4 & $(7),(1),(2)$ & 5 & 6 \\
$\tilde{R}_{5}$ & $(8)$ & 7 & $(8),(2),(1)$ & 6 & $(8),(2),(1)$ & 5 & $(8),(2),(1)$ & 3 & 4 \\
$\tilde{R}_{6}$ & $(1)$ & 2 & $(1),(7),(8)$ & 5 & $(1),(7),(8)$ & 6 & $(1),(7),(8)$ & 3 & 4 \\
$\tilde{R}_{7}$ & $(6)$ & 5 & $(6),(4),(3)$ & 8 & $(6),(4),(3)$ & 7 & $(6),(4),(3)$ & 1 & 2 \\
$\tilde{R}_{8}$ & $(3)$ & 4 & $(3),(5),(6)$ & 7 & $(3),(5),(6)$ & 8 & $(3),(5),(6)$ & 1 & 2
\end{tabular}

To check that $(\tilde{N}, \tilde{R})$ does not contain a 3 -ring we need to show that no three agent set $N^{\prime}=$ $\left\{i_{1}, i_{2}, i_{3}\right\} \subseteq\{1,2,3,4,5,6,7,8\}$ induces a corresponding 3 -ring market $\left(N^{\prime}, \tilde{R}_{N^{\prime}}\right)$. There are $\left(\begin{array}{l}8 \\ 3\end{array}\right)=$ 56 such sets. In the following table we list all these 3 agent sets $N^{\prime}$ with a symbolic explanation why $N^{\prime}$ does not induce a 3-ring market. The symbolic explanation " $\pi_{x}$ " for $x \in\{a, b, c, d\}$ will mean that no 3-ring is induced because by anonymity $\left(N^{\prime}, \tilde{R}_{N^{\prime}}\right)$ is a reduced market of $\left(N^{\pi_{x}}, R^{\pi_{x}}\right)$ and the original market $(N, R)$ does not have a 3-ring. The symbolic explanation " $q P_{q} r$ " for $q, r \in N^{\prime}$ will mean that agents $q$ and $r$ will never be in a 3-ring together because one does not find the other acceptable. 


\begin{tabular}{|lc||lc||lc||lc|}
\hline set & explanation & set & explanation & set & explanation & set & explanation \\
\hline \hline$\{123\}$ & $\pi_{a}$ & $\{124\}$ & $\pi_{a}$ & $\{1,2,5\}$ & $\pi_{b}$ & $\{1,2,6\}$ & $\pi_{b}$ \\
\hline$\{1,2,7\}$ & $1 P_{1} 7$ & $\{1,2,8\}$ & $1 P_{1} 8$ & $\{1,3,4\}$ & $\pi_{a}$ & $\{1,3,5\}$ & $3 P_{3} 5$ \\
\hline$\{1,3,6\}$ & $3 P_{3} 6$ & $\{1,3,7\}$ & $1 P_{1} 7$ & $\{1,3,8\}$ & $1 P_{1} 8$ & $\{1,4,5\}$ & $4 P_{4} 5$ \\
\hline$\{1,4,6\}$ & $4 P_{4} 6$ & $\{1,4,7\}$ & $1 P_{1} 7$ & $\{1,4,8\}$ & $1 P_{1} 8$ & $\{1,5,6\}$ & $\pi_{b}$ \\
\hline$\{1,5,7\}$ & $1 P_{1} 7$ & $\{1,5,8\}$ & $1 P_{1} 8$ & $\{1,6,7\}$ & $1 P_{1} 7$ & $\{1,6,8\}$ & $1 P_{1} 8$ \\
\hline$\{1,7,8\}$ & $1 P_{1} 7$ & $\{2,3,4\}$ & $\pi_{a}$ & $\{2,3,5\}$ & $3 P_{3} 5$ & $\{2,3,6\}$ & $3 P_{3} 6$ \\
\hline$\{2,3,7\}$ & $2 P_{2} 7$ & $\{2,3,8\}$ & $2 P_{2} 8$ & $\{2,4,5\}$ & $4 P_{4} 5$ & $\{2,4,6\}$ & $4 P_{4} 6$ \\
\hline$\{2,4,7\}$ & $2 P_{2} 7$ & $\{2,4,8\}$ & $2 P_{2} 8$ & $\{2,5,6\}$ & $\pi_{b}$ & $\{2,5,7\}$ & $2 P_{2} 7$ \\
\hline$\{2,5,8\}$ & $2 P_{2} 8$ & $\{2,6,7\}$ & $2 P_{2} 7$ & $\{2,6,8\}$ & $2 P_{2} 8$ & $\{2,7,8\}$ & $2 P_{2} 7$ \\
\hline$\{3,4,5\}$ & $3 P_{3} 5$ & $\{3,4,6\}$ & $3 P_{3} 6$ & $\{3,4,7\}$ & $\pi_{d}$ & $\{3,4,8\}$ & $\pi_{d}$ \\
\hline$\{3,5,6\}$ & $3 P_{3} 6$ & $\{3,5,7\}$ & $3 P_{3} 5$ & $\{3,5,8\}$ & $3 P_{3} 5$ & $\{3,6,7\}$ & $3 P_{3} 6$ \\
\hline$\{3,6,8\}$ & $3 P_{3} 6$ & $\{3,7,8\}$ & $\pi_{d}$ & $\{4,5,6\}$ & $4 P_{4} 5$ & $\{4,5,7\}$ & $4 P_{4} 5$ \\
\hline$\{4,5,8\}$ & $4 P_{4} 5$ & $\{4,6,7\}$ & $4 P_{4} 6$ & $\{4,6,8\}$ & $4 P_{4} 6$ & $\{4,7,8\}$ & $\pi_{d}$ \\
\hline$\{5,6,7\}$ & $\pi_{c}$ & $\{5,6,8\}$ & $\pi_{c}$ & $\{5,7,8\}$ & $\pi_{c}$ & $\{6,7,8\}$ & $\pi_{c}$ \\
\hline
\end{tabular}

Next, we show that for all $N^{\prime} \subseteq \tilde{N},\left|N^{\prime}\right|=2$, setting $M^{\prime}:=N^{\prime} \cup \tilde{\mu}\left(N^{\prime}\right)$, and the reduced market $\left(M^{\prime}, \tilde{R}_{M^{\prime}}\right), \tilde{\mu}_{M^{\prime}} \in \varphi\left(M^{\prime}, \tilde{R}_{M^{\prime}}\right)$. Note that $\left(M^{\prime}, \tilde{R}_{M^{\prime}}\right) \in \mathfrak{D}_{S N 3 R}$. There are $\left(\begin{array}{l}8 \\ 2\end{array}\right)=28$ sets $N^{\prime}$ to check.

1. $N^{\prime}=M^{\prime}=\{1,2\}$ :

$$
\begin{array}{l|ll}
\tilde{R}_{1} & 2 & 1 \\
\tilde{R}_{2} & 1 & 2
\end{array}
$$

Pareto optimality implies that $\tilde{\mu}_{M^{\prime}} \in \varphi\left(M^{\prime}, \tilde{R}_{M^{\prime}}\right)$.

2. $N^{\prime}=M^{\prime}=\{3,4\}$ :

$$
\begin{array}{l|ll}
\tilde{R}_{3} & 4 & 3 \\
\tilde{R}_{4} & 3 & 4
\end{array}
$$

Pareto optimality implies that $\tilde{\mu}_{M^{\prime}} \in \varphi\left(M^{\prime}, \tilde{R}_{M^{\prime}}\right)$.

3. $N^{\prime}=M^{\prime}=\{5,6\}$ :

$$
\begin{array}{l|ll}
\tilde{R}_{5} & 6 & 5 \\
\tilde{R}_{6} & 5 & 6
\end{array}
$$

Pareto optimality implies that $\tilde{\mu}_{M^{\prime}} \in \varphi\left(M^{\prime}, \tilde{R}_{M^{\prime}}\right)$.

4. $N^{\prime}=M^{\prime}=\{7,8\}$ :

$$
\begin{array}{l|ll}
\tilde{R}_{7} & 8 & 7 \\
\tilde{R}_{8} & 7 & 8
\end{array}
$$


Pareto optimality implies that $\tilde{\mu}_{M^{\prime}} \in \varphi\left(M^{\prime}, \tilde{R}_{M^{\prime}}\right)$.

5. . 8. $N^{\prime}=\{1,7\}, N^{\prime}=\{1,8\}, N^{\prime}=\{2,7\}$, or $N^{\prime}=\{2,8\}$ and $M^{\prime}=\{1,2,7,8\}$ :

$$
\begin{array}{l|llll}
\tilde{R}_{1} & 2 & 1 & 7 & 8 \\
\tilde{R}_{2} & 1 & 2 & 7 & 8 \\
\tilde{R}_{7} & 8 & 7 & 1 & 2 \\
\tilde{R}_{8} & 7 & 8 & 1 & 2
\end{array}
$$

Pareto optimality implies that $\tilde{\mu}_{M^{\prime}} \in \varphi\left(M^{\prime}, \tilde{R}_{M^{\prime}}\right)$.

9. .. 12. $N^{\prime}=\{3,5\}, N^{\prime}=\{3,6\}, N^{\prime}=\{4,5\}$, or $N^{\prime}=\{4,6\}$ and $M^{\prime}=\{3,4,5,6\}$ :

\begin{tabular}{l|llll}
$\tilde{R}_{3}$ & $(4)$ & 3 & 5 & 6 \\
$\tilde{R}_{4}$ & 3 & 4 & 5 & 6 \\
$\tilde{R}_{5}$ & 6 & 5 & 1 & 2 \\
$\tilde{R}_{6}$ & 5 & 6 & 1 & 2
\end{tabular}

Pareto optimality implies that $\tilde{\mu}_{M^{\prime}} \in \varphi\left(M^{\prime}, \tilde{R}_{M^{\prime}}\right)$.

13. ... 16. $N^{\prime}=\{1,3\}, N^{\prime}=\{1,4\}, N^{\prime}=\{2,3\}$, or $N^{\prime}=\{2,4\}$ and $M^{\prime}=\{1,2,3,4\}$ :

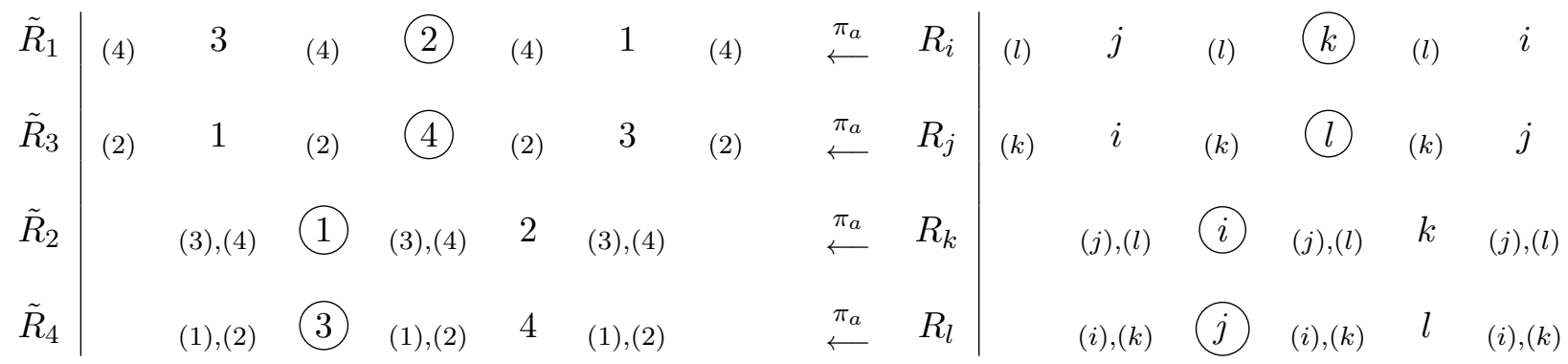

Note that $N^{\pi_{a}}=M^{\prime}$. In the above preference table, we have already indicated that $\tilde{R}_{M^{\prime}}=$ $R^{\pi_{a}}$. Then, by anonymity, $\tilde{\mu}_{M^{\prime}}=\mu^{\pi_{a}} \in \varphi\left(M^{\prime}, \tilde{R}_{M^{\prime}}\right)$.

17. .. 20. $N^{\prime}=\{1,5\}, N^{\prime}=\{1,6\}, N^{\prime}=\{2,5\}$, or $N^{\prime}=\{2,6\}$ and $M^{\prime}=\{1,2,5,6\}$ : 

\begin{tabular}{l|lll}
$\tilde{R}_{2}$ & (5) & 6
\end{tabular}
(1)
(5) 2
(5)
$\stackrel{\pi_{b}}{\longleftarrow} \quad R_{i} \mid(l) \quad j$
(l) $\quad$ (l) $\quad i \quad(l)$
$\tilde{R}_{6}$
(1) 2
(1)
(5)
(1) 6
(1)
$\stackrel{\pi_{b}}{\longleftarrow} R_{j}$
(k) $\quad i$
(k)
(l) $(k) j$
$(k)$
\begin{tabular}{l|ll}
$\tilde{R}_{1}$ & $(6),(5)$ & 2 \\
$\tilde{R}_{5}$ & $(2),(1)$ & 6
\end{tabular}
$\begin{array}{lllll}(6),(5) & 1 & (6),(5) & \stackrel{\pi_{b}}{ } & R_{k} \\ & & & & \\ (2),(1) & 5 & (2),(1) & \stackrel{\pi_{b}}{\longleftarrow} & R_{l}\end{array}$
$(j),(l)$
$(i),(k)$
$(j),(l) \quad k \quad(j),(l)$
$(i),(k) \quad l \quad(i),(k)$

Note that $N^{\pi_{b}}=M^{\prime}$. In the above preference table, we have already indicated that $\tilde{R}_{M^{\prime}}=$ $R^{\pi_{b}}$. Then, by anonymity, $\tilde{\mu}_{M^{\prime}}=\mu^{\pi_{b}} \in \varphi\left(M^{\prime}, \tilde{R}_{M^{\prime}}\right)$.

21. . 24. $N^{\prime}=\{3,7\}, N^{\prime}=\{3,8\}, N^{\prime}=\{4,7\}$, or $N^{\prime}=\{4,8\}$ and $M^{\prime}=\{3,4,7,8\}$ :
$\tilde{R}_{8}$
$\tilde{R}_{4}$
$\tilde{R}_{7}$
$\tilde{R}_{3}$
(7)
(3) 8
(3)
$\stackrel{\pi_{d}}{\longleftarrow} \quad R_{i}$
$R_{i} \quad(l) \quad j$
$(l)$
(k) $\quad i$
$(l)$

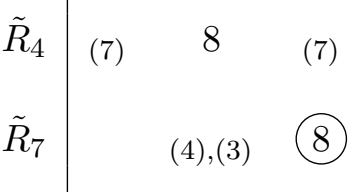
(3)
(7) 4
(7) $\stackrel{\pi_{d}}{\longleftarrow} R_{j}$
(k) $\quad i$
(k) (k) j
$(k)$
$\tilde{R}_{3} \quad$ (8),(7) (4)
$\begin{array}{lllll}(4),(3) & 7 & (4),(3) & \stackrel{\pi_{d}}{\longleftarrow} & R_{k} \\ & & & & \\ (8),(7) & 3 & (8),(7) & \stackrel{\pi_{d}}{\longleftarrow} & R_{l}\end{array}$
$(j),(l) \quad i$
$(i),(k)$
$(j),(l) \quad k \quad(j),(l)$
$(i),(k) \quad l \quad(i),(k)$

Note that $N^{\pi_{d}}=M^{\prime}$. In the above preference table, we have already indicated that $\tilde{R}_{M^{\prime}}=$ $R^{\pi_{d}}$. Then, by anonymity, $\tilde{\mu}_{M^{\prime}}=\mu^{\pi_{d}} \in \varphi\left(M^{\prime}, \tilde{R}_{M^{\prime}}\right)$.

25. .. 28. $N^{\prime}=\{5,7\}, N^{\prime}=\{5,8\}, N^{\prime}=\{6,7\}$, or $N^{\prime}=\{6,8\}$ and $M^{\prime}=\{5,6,7,8\}$ :
$\tilde{R}_{7}$
$\tilde{R}_{5}$
$\tilde{R}_{8}$
$\tilde{R}_{6}$
(8)
(6) 7
(3)
$\stackrel{\pi_{c}}{\longleftarrow} \quad R_{i} \mid(l)$
$j$
$(l)$
(k) $(l) \quad i$
$(l)$
(8) 7
(8) 6
(8) 5
(8) $\stackrel{\pi_{c}}{\longleftarrow} R_{j}$
(k) $\quad i$
(k)
(l) $(k) j$
$(k)$
\begin{tabular}{l|l}
$\tilde{R}_{8}$ & $(5),(6)$ \\
$\tilde{R}_{6}$ & $(7),(8)$
\end{tabular}
(7)
(5),(6) $\quad 8 \quad(5),(6)$
$\stackrel{\pi_{c}}{\longleftarrow} R_{k}$
${ }_{(j),(l)}$
${ }_{(i),(k)}$
$(j),(l) \quad k \quad(j),(l)$
(5) (7),(8) $6 \quad(7),(8)$
$\stackrel{\pi_{c}}{\longleftarrow} \quad R_{l}$
$(i),(k) \quad l \quad(i),(k)$

Note that $N^{\pi_{c}}=M^{\prime}$. In the above preference table, we have already indicated that $\tilde{R}_{M^{\prime}}=$ $R^{\pi_{c}}$. Then, by anonymity, $\tilde{\mu}_{M^{\prime}}=\mu^{\pi_{c}} \in \varphi\left(M^{\prime}, \tilde{R}_{M^{\prime}}\right)$.

We have shown that for all $N^{\prime} \subseteq \tilde{N},\left|N^{\prime}\right|=2$, setting $M^{\prime}:=N^{\prime} \cup \tilde{\mu}\left(N^{\prime}\right)$, and the reduced market $\left(M^{\prime}, \tilde{R}_{M^{\prime}}\right) \in \mathfrak{D}_{S N 3 R}, \tilde{\mu}_{M^{\prime}} \in \varphi\left(M^{\prime}, \tilde{R}_{M^{\prime}}\right)$. Hence, by converse consistency, $\tilde{\mu} \in \varphi(\tilde{N}, \tilde{R})$. However, note that matching $\{(1,3),(2,6),(4,8),(5,7)\}$ is strictly preferred by everybody; a contradiction to Pareto optimality.

Cases 1, 2, and 3 have now all resulted in contradictions. Recall that these three cases resulted from the assumption that there exists a solvable market without 3 -rings $(\bar{N}, \bar{R}) \in \mathfrak{D}_{S N 3 R}$ and a matching $\bar{\mu} \in \varphi(\bar{N}, \bar{R})$ with a blocking pair $\{i, j\}$ for $\bar{\mu}$. Hence, we have now shown that $\varphi \subseteq$ core. 


\section{B Independence of Properties in Theorem 1}

For each of the examples introduced to establish independence below we indicate the property of Theorem 1 it fails (while it satisfies all remaining properties). All solutions in this section are defined on $\mathfrak{D}_{S N 3 R}$.

Example (Not Pareto Optimal). The solution $I R$ assigns the set of individually rational matchings.

Example (Not Conversely Consistent). The solution $P O \cap I R$ assigns the intersection of the Pareto solution $P O$ with solution $I R$.

To see that converse consistency is violated, consider the following market $(N, R) \in \mathfrak{D}_{S N 3 R}$ with $N=\{1,2,3,4,5,6\}$ and matching $\mu=\{(1,2),(3,4),(5,6)\}$ :

\begin{tabular}{l|llll}
$R_{1}$ & 4 & 2 & 1 & $\ldots$ \\
$R_{2}$ & 5 & 1 & 2 & $\ldots$ \\
$R_{3}$ & 6 & 4 & 3 & $\ldots$ \\
$R_{4}$ & 1 & 3 & 4 & $\ldots$ \\
$R_{5}$ & 2 & 6 & 5 & $\ldots$ \\
$R_{6}$ & 3 & 5 & 6 & $\ldots$
\end{tabular}

It is easy to check that converse consistency would imply that $\mu \in P O \cap I R$ even though all agents prefer matching $\{(1,4),(2,5),(3,6)\}$.

Example (Not Consistent). The solution $\zeta$ for any market $(N, R) \in \mathfrak{D}_{S N 3 R}$ such that $|N| \leq 4$ assigns the core and otherwise equals $P O \cap I R$.

Example (Not Individually Rational). We define a subsolution of the Pareto solution $\psi \varsubsetneqq P O$ by eliminating all matchings that have a blocking pair composed of two different agents, i.e., in difference to the core this solution allows for degenerate blocking pairs of the form $\{i, i\}$.

Example (Not Anonymous). We define solution $\xi$ as follows. First, consider the following market $(\hat{N}, \hat{R}) \in \mathfrak{D}_{S N 3 R}$ with $\hat{N}=\{1,2,3\}$ and matching $\hat{\mu}=\{(1,3), 2\}$ :

$$
\begin{array}{c|ccc}
\hat{R}_{1} & 2 & 3 & 1 \\
\hat{R}_{2} & 1 & 2 & 3 \\
\hat{R}_{3} & 1 & 3 & 2
\end{array}
$$

The unique stable matching for this market is $\{(1,2), 3\}$ and solution $\xi$ adds matching $\hat{\mu}$ to the core, i.e., $\xi(\hat{N}, \hat{R})=\operatorname{core}(\hat{N}, \hat{R}) \cup\{\hat{\mu}\}$.

Next, consider roommates markets $(N, R) \in \mathfrak{D}_{S N 3 R}$ such that $\hat{N} \subseteq N, R_{\hat{N}}=\hat{R}$ and let $\bar{\mu} \in \operatorname{core}(N, R)$ such that $\bar{\mu}_{\hat{N}}=\{(1,2), 3\}$ and at matching $\tilde{\mu}=\bar{\mu}_{N \backslash \hat{N}} \cup \hat{\mu},\{1,2\}$ is the unique blocking pair. Then, solution $\xi$ adds the unstable matching $\tilde{\mu}$ to $\operatorname{core}(N, R)$. Note that solution $\xi$ might add several of these additional unstable matchings that replace the component $\{(1,2), 3\}$ in a stable matching with component $\hat{\mu}$. For all remaining roommates markets $(N, R) \in \mathfrak{D}_{S N 3 R}$, $\xi(N, R)=\operatorname{core}(N, R)$. Note that the addition of matchings with the "component" $\hat{\mu}$ is designed such that $\xi$ satisfies consistency and converse consistency. 


\section{Proof of Theorem 2}

The proof of Theorem 2 follows the same arguments as the proof of Theorem 1 . Proposition 1 and Lemma 1 hold on the marriage market domain $\mathfrak{D}_{M}$. Furthermore, marriage markets are solvable and cannot contain 3-rings and therefore we can basically just replicate the arguments in the proof of Theorem 1. The only steps in the proof that require special "marriage market attention" are in the Uniqueness Part I (see Appendix A) when it has to be confirmed that the constructed market $(\tilde{N}, \tilde{R})$ contains no 3-ring. The corresponding steps for marriage markets follow.

Case $1(|N|=2)$ remains the same.

Case $2(|N|=3)$ : there is only one possible marriage market $(N, R)$

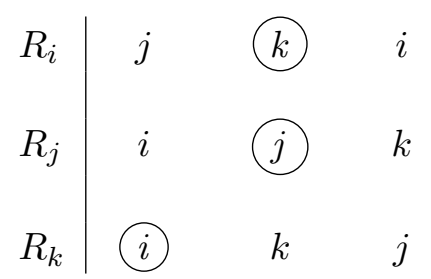

and one constructed market $(\tilde{N}, \tilde{R})$

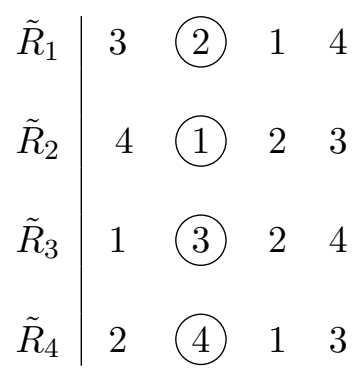

Note that $(\tilde{N}, \tilde{R}) \in \mathfrak{D}_{M}$, e.g. with $M=\{1,4\}$ and $W=\{2,3\}$.

Case $3(|N|=4)$ : there are only 16 possible marriage markets $(N, R)$

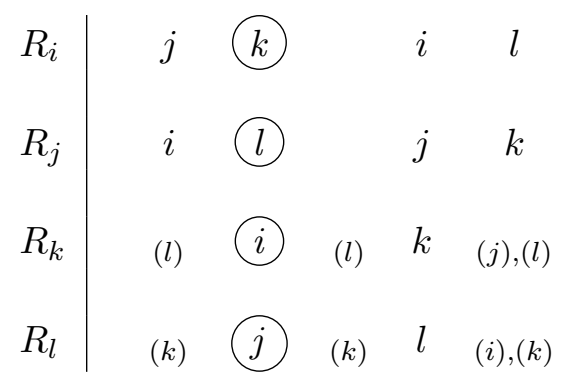

and the following constructed market(s) $(\tilde{N}, \tilde{R})$ 


\begin{tabular}{l|llllllll}
$\tilde{R}_{1}$ & 3 & $(5)$ & 2 & $(5)$ & 1 & $(4),(6),(5)$ & 7 & 8 \\
$\tilde{R}_{2}$ & 6 & $(4)$ & 1 & $(4)$ & 2 & $(5),(3),(4)$ & 7 & 8 \\
$\tilde{R}_{3}$ & 1 & $(7)$ & 4 & $(7)$ & 3 & $(2),(8),(7)$ & 5 & 6 \\
$\tilde{R}_{4}$ & 8 & $(2)$ & 3 & $(2)$ & 4 & $(7),(1),(2)$ & 5 & 6 \\
$\tilde{R}_{5}$ & 7 & $(1)$ & 6 & $(1)$ & 5 & $(8),(2),(1)$ & 3 & 4 \\
$\tilde{R}_{6}$ & 2 & $(8)$ & 5 & $(8)$ & 6 & $(1),(7),(8)$ & 3 & 4 \\
$\tilde{R}_{7}$ & 5 & $(3)$ & 8 & $(3)$ & 7 & $(6),(4),(3)$ & 1 & 2 \\
$\tilde{R}_{8}$ & 4 & $(6)$ & 7 & $(6)$ & 8 & $(3),(5),(6)$ & 1 & 2
\end{tabular}

Note that $(\tilde{N}, \tilde{R}) \in \mathfrak{D}_{M}$, e.g. with $M=\{1,4,6,7\}$ and $W=\{2,3,5,8\}$.

\section{References}

Bartholdi, J. J., III and Trick, M. A. (1986): "Stable Matching with Preferences Derived from a Psychological Model." Operations Research Letters, 5: 156-169.

Becker, G. S. (1973): “A Theory of Marriage: Part I.” Journal of Political Economy, 81: 813-846.

Can, B. and Klaus, B. (2013): "Consistency and Population Sensitivity Properties in Marriage and Roommate Markets." Social Choice and Welfare, 41: 835-862.

Chung, K.-S. (2000): "On the Existence of Stable Roommate Matchings." Games and Economic Behavior, 33: 206-230.

Demange, G. and Wooders, M., editors (2004): Group Formation in Economics; Networks, Clubs and Coalitions. Cambridge University Press, Cambridge.

Gale, D. and Shapley, L. S. (1962): "College Admissions and the Stability of Marriage." American Mathematical Monthly, 69: 9-15.

Inada, K. I. (1964): “A Note on the Simple Majority Decision Rule." Econometrica, 32: 525-531.

Inada, K. I. (1969): “The Simple Majority Decision Rule.” Econometrica, 37: 490-506.

Jackson, M. O. (2008): Social and Economic Networks. Princeton University Press, Princeton.

Klaus, B. (2011): "Competition and Resource Sensitivity in Marriage and Roommate Markets." Games and Economic Behavior, 72: 172-186.

Klaus, B. (2017): "Consistency and its Converse for Roommate Markets." Cahier de recherches économiques du DEEP No. 17.03 (https://ideas.repec.org/p/lau/crdeep/17.03.html). 
Knuth, D. (1997): Stable Marriage and its Relation to other Combinatorial Problems: An Introduction to the Mathematical Analysis of Algorithms. American Mathematical Society.

Nizamogullari, D. and Özkal-Sanver, İ. (2014): "Characterization of the Core in Full Domain Marriage Problems." Mathematical Social Sciences, 69: 34-42.

Özkal-Sanver, İ. (2004): “A Note on Gender Fairness in Matching Problems." Mathematical Social Sciences, 47: 211-217.

Özkal-Sanver, İ. (2010): "Impossibilities in Roommates Problems." Mathematical Social Sciences, 59: $360-363$.

Roth, A. E. and Sotomayor, M. A. O. (1990): Two-Sided Matching: A Study in Game-Theoretic Modeling and Analysis. Cambridge University Press, Cambridge.

Sasaki, H. and Toda, M. (1992): "Consistency and Characterization of the Core of Two-Sided Matching Problems." Journal of Economic Theory, 56: 218-227.

Sen, A. K. and Pattanaik, P. K. (1969): "Necessary and Sufficient Conditions for Rational Choice under Majority Decision." Journal of Economic Theory, 1: 178-202.

Thomson, W. (2015): Consistent Allocation Rules. Cambridge University Press. Forthcoming.

Toda, M. (2006): "Monotonicity and Consistency in Matching Markets." International Journal of Game Theory, 34: 13-31. 